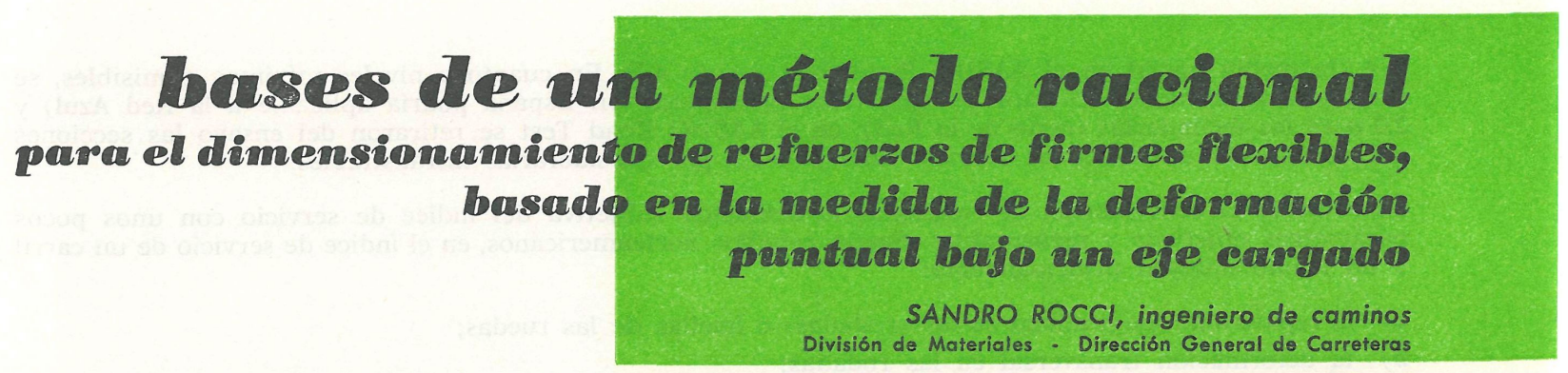

514.54

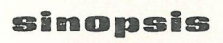

Este artículo constituye una descripción cualitativa de los factores que intervienen en el com. portamiento del firme de una carretera, y de las relaciones entre ellos, estableciéndose un esquema funcional cuyo objetivo último económico es la minimización del coste total (construc. ción + conservación + explotación) de una carretera. Al mismo tiempo, se establecen las bases para un método de dimensionamiento de refuerzo de firmes flexibles, cuya justificación cuantitativa será objeto de un próximo artículo.

\title{
Imaำ
}

El paso del tiempo (y por consiguiente de un tráfico pesado) sobre el firme flexible de una carretera nueva produce un deterioro progresivo del mismo, hasta que, al cabo de un cierto tiempo (llamado período de proyecto del firme nuevo), llega un momento en que se hace preciso restablecer unas buenas condiciones de vialidad: generalmente las iniciales. Esto podría llevarse a cabo realizando un acondicionamiento superficial; pero como su valor estructural es prácticamente nulo, al cabo de un tiempo relativamente breve el deterioro del firme acondicionado habría progresado de nuevo hasta un nivel inadmisible. Se hace preciso, por lo tanto, que al cabo del primer ciclo de deterioro se proceda a un refuerzo del firme flexible, que cumpla dos misiones simultáneas:

1." Restablecer las condiciones de vialidad de un pavimento nuevo.

2. ${ }^{2}$ Hacer que el deterioro del firme reforzado progrese a un ritmo lo suficientemente lento como para que, hasta que se alcance de nuevo el nivel inadmisible, haya transcurrido como mínimo un cierto tiempo, denominado periodo de proyecto del refuerzo (fig. 1).

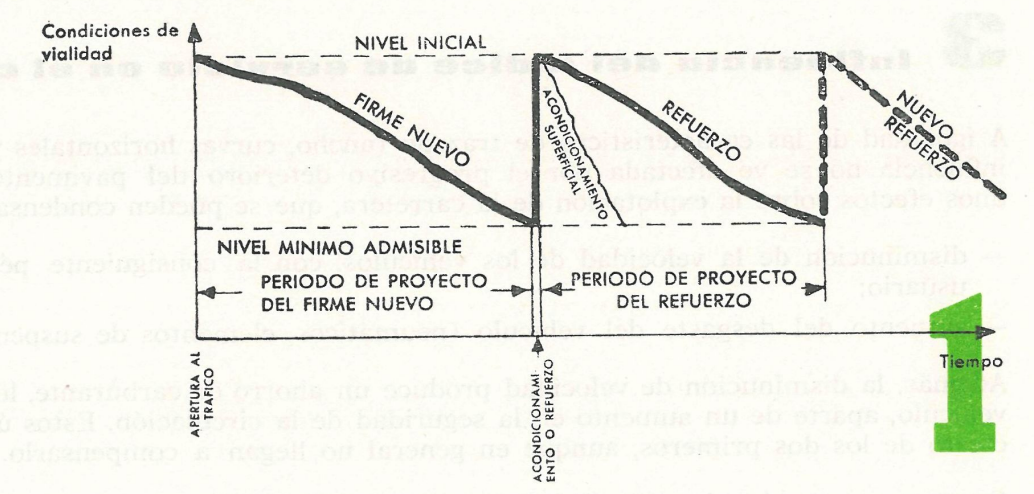

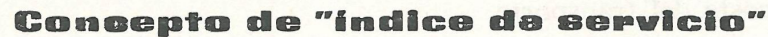

Las condiciones de vialidad, que son las que interesan al usuario de la carretera, es conveniente resumirlas en un solo parámetro denominado eíndice de servicio" $(p)$. Para el AASHO Road Test se desarrolló este concepto, puramente subjetivo del usuario, clasificándolo así:

\begin{tabular}{|c|c|}
\hline Condiciones de vialidad & $p$ \\
\hline $\begin{array}{lllllllllllll}\text { Excelentes } & \ldots & \ldots & \ldots & \ldots & \ldots & \ldots & \ldots & \ldots & \ldots & \ldots & \ldots & \ldots\end{array}$ & 5 a 4 \\
\hline $\begin{array}{llllllllllllll}\text { Buenas } & \ldots & \ldots & \ldots & \ldots & \ldots & \ldots & \ldots & \ldots & \ldots & \ldots & \ldots & \ldots & \ldots\end{array}$ & 4 a 3 \\
\hline $\begin{array}{llllllllllll}\text { Regulares } & \ldots & \ldots & \ldots & \ldots & \ldots & \ldots & \ldots & \ldots & \ldots & \ldots & \ldots\end{array} \ldots$ & 3 a 2 \\
\hline $\begin{array}{llllllllllllll}\text { Malas } & \ldots & \ldots & \ldots & \ldots & \ldots & \ldots & \ldots & \ldots & \ldots & \ldots & \ldots & \ldots & \ldots\end{array}$ & 2 a 1 \\
\hline $\begin{array}{lllllllllllll}\text { Pésimas } & \ldots & \ldots & \ldots & \ldots & \ldots & \ldots & \ldots & \ldots & \ldots & \ldots & \ldots & \ldots\end{array}$ & 1 a 0 \\
\hline
\end{tabular}

La determinación del índice de servicio se realizó, en principio, por un grupo de usuarios convenientemente seleccionado, quien también determinó si las condiciones de vialidad eran aceptables o no. Una carretera nueva, o recién acondicionada o reforzada, debe presentar un índice de servicio inicial $p_{o}$ superior 
a 4 (la media inicial en el AASHO Road Test fue de 4,2). En cuanto a niveles mínimos admisibles, se preconiza actualmente 2,5 para las carreteras principales (en España podría apliccis ae a la Red Azul) y 2,0 para las secundarias (resto de la Red); en el AASHO Road Test se retiraron del ensayo las secciones que alcanzaron un índice de servicio final $p_{f}=1,5$, por considerarlas intransitables.

Es evidente la conveniencia de relacionar la definición subjetiva del índice de servicio con unos pocos parámetros objetivos y mensurables. Según estudios norteamericanos, en el índice de servicio de un carril $\mathrm{y}$ un tramo influyen principalmente:

a) la regularidad longitudinal de las «rodadas» o huellas de las ruedas;

b) la deformación transversal en las rodadas;

c) la proporción de la superficie del pavimento que exhibe deterioros iguales o peores que las fisuraciones reticulares, o reparaciones por conservación normal.

Los factores a) y $b$ ) se promedian para las dos rodadas que corresponden a un carril. En la realidad, el carril exterior, como se ve más solicitado por los vehículos pesados, es el que exhibe un índice de servicio más bajo.

Un hecho que hay que resaltar es que el índice de servicio no es un concepto puntual, sino que se refiere a una cierta longitud de un carril. En el AASHO Road Test, dicha longitud correspondía a una sección de ensayo cuyas características estructurales eran homogéneas. En las aplicaciones prácticas (Tramos de Control y subsiguiente dimensionamiento de refuerzos) en las que se desconocen, por lo general, las características estructurales del firme existente, la definición de la longitud a la que hay que referir el índice de servicio es un problema ya más complejo, puesto que hay que determinar, dentro del Tramo de Control (en cuya definición no entran consideraciones estructurales), unos subtramos en los que las características estructurales puedan considerarse homogéneas. Para ello se verá más adelante que, a falta de un inventario completo (del que generalmente es difícil disponer), es interesante que estas características estructurales puedan representarse por un parámetro global mensurable (por ejemplo la deformación puntual elástica).

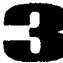

A igualdad de las características de trazado (ancho, curvas horizontales y pendientes longitudinales), cuya influencia no se ve afectada por el progresivo deterioro del pavimento de la carretera, éste produce unos efectos sobre la explotación de la carretera, que se pueden condensar en dos conceptos:

- disminución de la velocidad de los vehículos, con la consiguiente pérdida de tiempo por parte del usuario;

- aumento del desgaste del vehículo (neumáticos, elementos de suspensión, etc.).

Además, la disminución de velocidad produce un ahorro de carburante, lubricante y un menor desgaste del vehículo, aparte de un aumento en la seguridad de la circulación. Estos últimos factores se contraponen al efecto de los dos primeros, aunque en general no llegan a compensarlo.

Como consecuencia, el progresivo deterioro de una carretera con el tiempo produce un aumento correlativo del coste del transporte; y el restablecimiento de un índice de servicio adecuado, por acondicionamiento superficial o por refuerzo, disminuye dicho coste del transporte.

\subsection{VARIACION DE LA VELOCIDAD SEgUN EL ESTADO DEL PAVIMENTo}

Se puede distinguir, para analizar las causas que motivan la elección de la velocidad en un tramo de carretera, entre vehículos ligeros (turismos) y vehículos pesados (camiones y autobuses).

\subsubsection{Vehículos ligeros}

En la velocidad adoptada por los vehículos ligeros, influyen primordialmente:

7. la velocidad que el conductor estima como "deseable», y en cuya elección interviene subjetivamente la valoración de su propio tiempo y del gasto del vehículo, el objeto del viaje, la satisfacción proporcionada por la velocidad, etc.; 
1 las características de la carretera: capacidad, visibilidad, pendientes longitudinales, curvas horizontales, estado del pavimento, etc. A este respecto puede distinguirse entre condiciones de «circulación libre» (capacidad de la carretera suficiente para la intensidad de tráfico que soporta, visibilidad suficiente para no impedir cualquier maniobra de paso, rasante llana, recta, pavimento en buen estado), en la que cada conductor puede circular a su velocidad deseada, y las condiciones de "circulación restringida» que la alteración de una o varias de estas características pueden imponer, y que tienen como consecuencia la disminución de la velocidad;

F las posibilidades del vehículo: potencia, velocidad máxima, consumo, etc.

Cuando la coyuntura del mercado del automóvil lo permite, todo usuario tiende a adquirir un vehículo tal, que su velocidad en condiciones de circulación libre se aproxima lo más posible a la deseada por él. La función global de distribución de velocidades en condiciones de libre circulación es la suma ponderada de las funciones de distribución de velocidades de cada tipo de vehículo ligero y, por lo tanto, en ella influye la composición del tráfico ligero (proporción de los vehículos ligeros según su tipo), y las velocidades deseadas por los usuarios de cada tipo de vehículo ligero. A medida que se van introduciendo condiciones restrictivas en la circulación, las velocidades reales de los vehículos se alejan de las deseadas por sus conductores, y la función global de distribución de velocidades se altera. Esta alteración se traduce en una disminución de la velocidad media y en una deformación, habiéndose podido comprobar que la deformación es independiente de la causa de la restricción en la circulación; lo que equivale a decir que las funciones globales de distribución de velocidades pueden representarse adecuadamente por un solo parámetro (la velocidad media), ya que a cada velocidad media le corresponde una forma de distribución única.

La acción de las diversas condiciones restrictivas de la circulación no es aditiva, sino que la velocidad media se adapta a la condición restrictiva más desfavorable. El empeoramiento del estado de vialidad del pavimento (medido por la disminución del índice de servicio) está ligado con una disminución de la velocidad media de los vehículos ligeros que transitan por el tramo.

\subsubsection{Vehículos pesados}

En la velocidad adoptada por los vehículos pesados influyen las mismas causas que en los vehículos ligeros, pero con ciertos matices peculiares de la naturaleza industrial de los vehículos pesados. Así, en la elección de la velocidad deseable influyen mucho más las consideraciones de explotación económica del vehículo que la valoración subjetiva del tiempo del conductor; y en las posibilidades del vehículo para desarrollar ciertas velocidades interviene de forma decisiva su estado de carga.

La distribución de velocidades de los vehículos pesados está mucho menos estudiada que la de los vehículos ligeros.

\subsection{TIEMPO PERDIDO POR DISMINUCION DE VELOCIDAD}

El tiempo que tarde un determinado vehículo en recorrer un tramo se puede dividir en tres tipos: tiempo de recorrido, tiempo de parada y tiempo de espera.

El tiempo de espera corresponde a los períodos en que el vehículo se halla detenido, por ejemplo por un semáforo o paso a nivel. El tiempo de parada corresponde a las maniobras de aumento o disminución de velocidad, para adaptar ésta a distintas condiciones restrictivas de la circulación; y, salvo casos especiales, se considera englobado en el tiempo de recorrido.

El tiempo que un vehículo ligero invierte en el recorrido de un tramo tiene un valor que es función de las características del usuario: su renta, el objeto del viaje, la satisfacción relacionada con la velocidad, etcétera. Para llevar a cabo una clasificación de usuarios con este fin, se puede recurrir a fijar el valor del tiempo en función de la velocidad que ellos estimen como deseable; hallándose que, cuanto mayor es esta velocidad deseable, mayor es también el valor de la unidad de tiempo. Por ello, el deterioro del pavimento, al producir una disminución de la velocidad de los vehículos ligeros que transitan por la carretera, se traduce en un aumento del tiempo de recorrido y, por tanto, del coste del transporte ligero.

La valoración del tiempo que invierte un vehículo pesado en recorrer un tramo es más difícil, ya que intervienen factores dependientes de la explotación del vehículo, principalmente de la longitud del viaje y del estado de carga. En viajes cortos reiterados, el disminuir el tiempo de recorrido puede permitir realizar más viajes por jornada, mientras que en un viaje largo que ocupa una jornada entera, la disminución de tiempo de recorrido no supone una mejor explotación del vehículo. 


\subsection{VARIACION DEL COSTE DE FUNCIONAMIENTO}

\subsubsection{Consumo de carburantes}

El consumo de carburantes de un vehículo dado depende, fundamentalmente, de su velocidad. Además de este factor, interviene en menor grado la pendiente longitudinal del camino (que puede obligar incluso a cambiar de marcha, lo que implica un aumento del consumo). En los vehículos pesados, la influencia del estado de carga es muy importante.

La disminución de velocidad consecuente del deterioro del pavimento se traduce en un menor consumo de carburante, lo que implica un cierto ahorro.

\subsubsection{Consumo de lubricantes}

Se puede admitir que el consumo de lubricantes es proporcional al consumo de carburantes.

\subsubsection{Desgaste del vehículo}

Determinadas piezas del vehículo, entre las que destacan los neumáticos y los órganos de suspensión, tienen un desgaste directo tanto más acusado cuanto peor es el estado de vialidad del camino. También interviene en el desgaste la velocidad del vehículo.

\subsubsection{Amortización y varios}

Los gastos de amortización del vehículo, seguros, impuestos, etc., se suelen calcular como una tasa fija por kilómetro recorrido. Existe una forma de desgaste indirecto, consecuencia del estado de vialidad del camino, que se refleja en una disminución del número de kilómetros que se toma como base para calcular la amortización y demás gastos, disminución tanto mayor cuanto peor es dicho estado.

\subsection{SEGURIDAD EN LA CIRCULACION}

Determinados accidentes son consecuencia de la velocidad. Por lo tanto, la disminución de velocidad como consecuencia del deterioro del pavimento tiene, indirectamente, un efecto favorable en la disminución del número de accidentes. Esta consideración es difícil de tener en cuenta, tanto más porque las obras de refuerzo generalmente se combinan con mejoras locales que pretenden eliminar las causas de los accidentes (puntos negros).

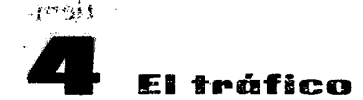

La solicitación más importante a que se ven sometidos los firmes es la que procede de la acción de las cargas transmitidas por el tráfico al pavimento. Como unidad para su evaluación se adopta el eje (en su acepción estructural que se define a continuación), caracterizado por su tipo y carga.

Comoquiera que la mayoría de los estudios de tráfico se refieren a los vehículos y no a los ejes, se hace necesario establecer los criterios de paso de uno a otro concepto.

\subsection{TIPOS DE RUEDAS, EJES Y VEHICULOS}

1. La rueda es el dispositivo que transmite las cargas al pavimento. Puede ser sencilla o gemela (formada por dos sencillas adyacentes, de forma que la separación entre ellas sea inferior al espesor de una de ellas). Excepcionalmente se podrán presentar ruedas múltiples, formadas por un conjunto de más de dos ruedas sencillas, en el que la separación entre dos adyacentes no sea superior al diámetro de las mismas. 
2. El eje puede tener dos acepciones:

a) el eje físico está situado transversalmente al vehículo, y en él van montadas dos ruedas sencillas o gemelas (una a cada lado), o una rueda múltiple. Cada «eje físico» da origen a una "cuenta» de los dispositivos aforadores automáticos;

b) el eje estructural es un conjunto de ejes físicos que están lo suficientemente próximos entre sí (separación entre dos consecutivos inferior a $2,6 \mathrm{~m}$ ) para que su acción sobre el firme sea conjunta. El eje estructural puede ser, por tanto, sencillo, doble o múltiple. Normalmente todas las ruedas de un eje estructural son de igual naturaleza, y la carga del mismo se reparte por igual entre los ejes físicos que lo componen.

3. Vehiculo es un conjunto de ejes estructurales que obedece a un mismo conductor. Por consiguiente, se requiere una traducción de los aforos automáticos (que cuentan ejes físicos) para conocer el número de vehículos y el de ejes estructurales transitados.

\subsection{ANALISIS DEL PASO DE UN VEHICULO POR LA CARRETERA}

Supóngase un tramo «i» de una carretera, cuyo ancho es de un carril y en cuya longitud $l_{i}$ se pueden considerar homogéneas las características estructurales; el índice de servicio de este tramo en el instante «t»será $p_{i t}$.

El paso de un vehículo « $j$ » en el instante «t» por el tramo «i» tiene una doble consecuencia:

a) el coste de explotación del vehículo al pasar sobre el tramo es:

$$
e_{i j t}=l_{i} \times c_{j}\left(i, p_{i t}\right),
$$

siendo $c_{j}$ el coste, por unidad de longitud, de explotación del vehículo «j» sobre un tramo cuyas características de trazado sean las del tramo «i» $\mathrm{y}$ cuyo índice de servicio sea $p_{i t}$;

b) por el tramo pasan $\operatorname{los} n_{j}$ ejes estructurales del vehículo «j», cada uno de los cuales pertenece a un determinado tipo y tiene una cierta carga.

Cada eje produce una deformación en el pavimento (prácticamente recuperable en su totalidad), cuya magnitud depende del tipo y carga del eje, de la velocidad del vehículo, y de las condiciones estructurales de la carretera. En el AASHO Road Test se puso de manifiesto que esta deformación está asociada a una disminución irreversible del índice de servicio del tramo, que por lo tanto es acumulativa a las producidas por el paso de otros vehículos.

Una simplificación muy importante la constituye el hecho de que tanto la deformación del pavimento como la disminución del índice de servicio producidas por el paso de un vehículo ligero son despreciables, comparadas con las producidas por el tráfico pesado. Por lo tanto, se puede admitir que $p_{i t}$ no se modifica por el paso de un vehículo ligero.

\subsection{ANALISIS DEL TRAFICO}

Extendiendo el análisis anterior, para contabilizar por un lado los costes de explotación de la carretera, y por otro la disminución acumulativa de su índice de servicio, haría falta en rigor conocer, para cada vehículo que transita por

la misma (fig. 2):

- su tipo (amortización, consumo, etc.);

- el tipo y carga de cada uno de sus ejes estructurales;

- su velocidad;

- el instante de su paso.

Naturalmente, es prácticamente imposible disponer de tal abundancia de datos en su totalidad, por lo que se recurre, en general, al muestreo y generalización, y a manejar cifras medias.

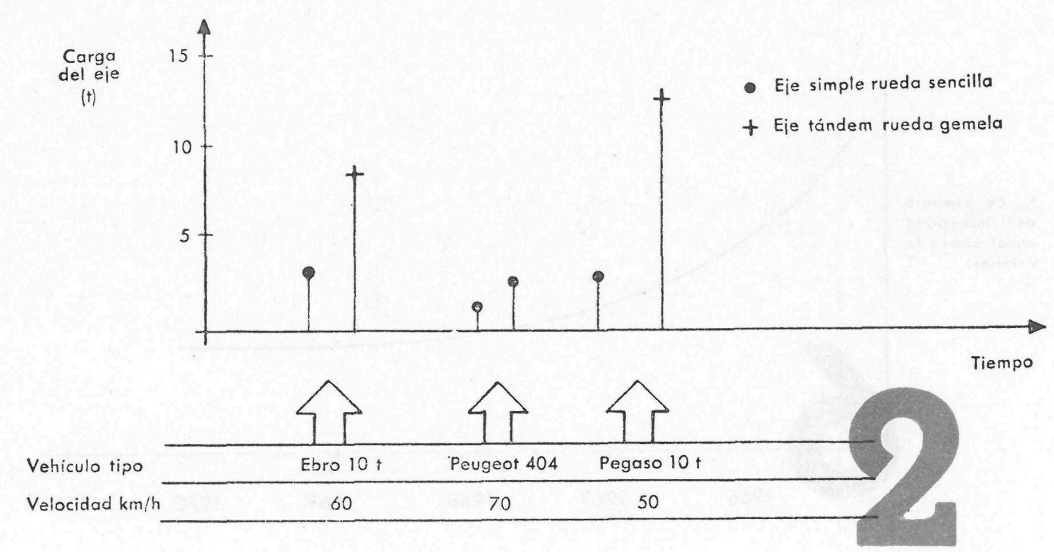




\subsubsection{Intensidad de tráfico}

El tráfico total puede definirse por su intensidad. Esta es fundamentalmente variable según la hora del día, el día de la semana, la semana del año y el año.'
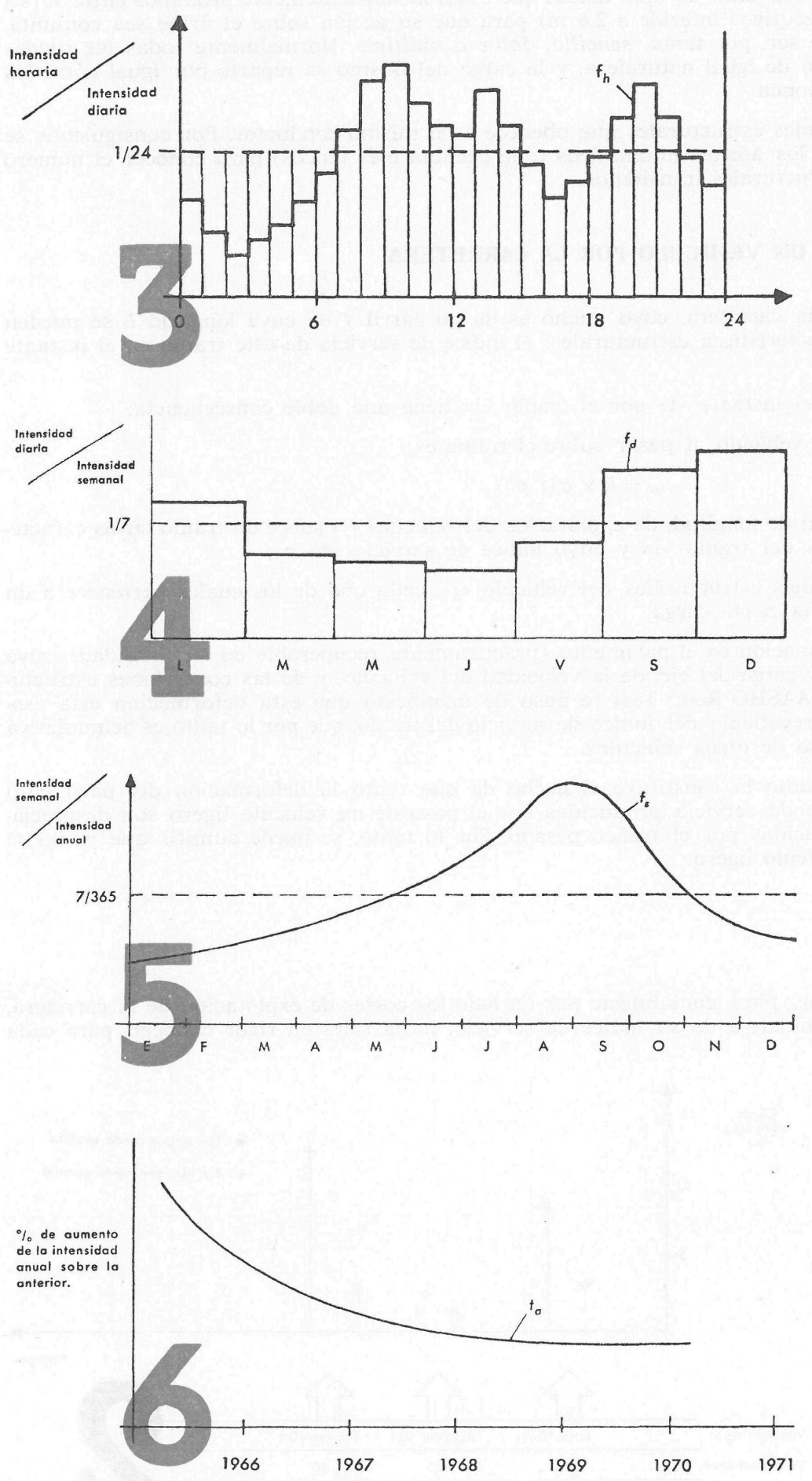

La intensidad horaria real se puede referir a la diaria por una funcíón de distribución $f_{h}$ (fig. 3 ).

A no ser que existan motivos concretos para afirmar lo contrario, se puede admitir que esta función de distribución $f_{h}$ es independiente del día a que se refiere (o sea, que no existen variaciones diarias ni estacionales, ni evolución anual).

La intensidad diaria se puede referir a la semanal por una función de distribución $f_{d}$ (fig. 4).

Igualmente $\mathrm{y}$, salvo justificación en contra, se puede admitir que la función de distribución $f_{d}$ es independiente de la semana a que se refiere, o sea, que no existen variaciones estacionales ni anuales.

$\mathrm{La}$ intensidad semanal se puede referir a la anual por una función de distribución $f_{s}$ (fig. 5).

Asimismo y, salvo justificación en contrario, se puede admitir que la función de distribución $f_{s}$ es independiente del año a que se refiere, es decir, que no existe variación anual.

$\mathrm{La}$ intensidad anual (igual a $365 \times$ la intensidad media diaria IMD, que es la que se maneja habitualmente) crece a lo largo de los años con una ley de interés compuesto, cuya tasa $t_{a}$ está ligada a la de crecimiento del parque e índice de motorización de la zona, y que generalmente es decreciente (fig. 6).

Las cuatro funciones $f_{h}, f_{d}$, $f_{s}$ y $t_{a}$, así como la IMD en un año cualquiera, definen perfectamente la intensidad de tráfico en cualquier instante: la dificultad estriba solamente en la definición de dichas funciones, que dependen fundamentalmente del tipo de servicio que presta la carretera. 


\subsubsection{Clasificación de vehículos}

En una intensidad de tráfico de las estudiadas en el epígrafe anterior es poco frecuente distinguir los tipos de vehículos que la integran, aunque ello es perfectamente posible en estudios particulares. Unicamente es habitual dar el porcentaje de vehículos pesados, referido a la IMD (o sea, a la intensidad anual). Este porcentaje se suele admitir (salvo justificación en contrario) que se puede aplicar también a las funciones de distribución $f_{h}, f_{d}$ y $f_{s}$; también se puede fijar su evolución anual (función del índice de motorización), que se admite es decreciente (fig. 7).

Para otras clasificaciones de vehículos (relacionadas con el estudio del coste del transporte) se suele recurrir a generalizar estudios particulares.

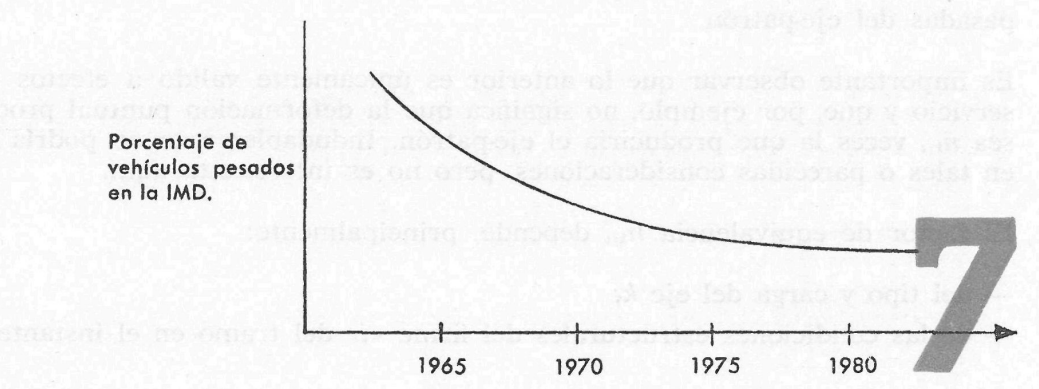

\subsubsection{Clasificación de los vehículos pesados por tipos de ejes}

Ciñéndose ya tan sólo al tráfico de vehículos pesados, el número de ejes estructurales de cada tipo que a ellos corresponde depende de la composición del parque de vehículos pesados. También en este caso lo más que se suele hacer es generalizar estudios particulares. En el momento actual en España estos estudios indican que cada vehículo pesado da origen a 2 ejes estructurales; que de éstos más del 95 por 100 son simples; y que de este 95 por 100 la mitad tienen ruedas sencillas (ejes delanteros), y la otra mitad, gemelas (ejes traseros); la incidencia de otros tipos de ejes es muy pequeña. No se suelen considerar variaciones horarias, diarias, estacionales ni anuales, salvo casos en que se tengan motivos concretos para fundamentar dicha consideración.

\subsubsection{Clasificación de los ejes estructurales según su carga}

La carga de un determinado eje estructural de un vehículo pesado se compone de dos partes: la debida a la tara y la debida a la carga útil. Ambas dependen del tipo de vehículo, y la segunda, además, del estado de carga (vacío o lleno); aunque mejor seríahablar de «estado de sobrecarga», puesto que en el momento actual en España la proporción de ejes que van sobrecargados por encima del límite legal es muy elevado.

Aunque para estudios especiales puede interesar estudiar por separado la tara y la carga útil, y relacionar la carga debida a esta última con el estado de carga, los aforos de pesaje suelen proporcionar como resultado una distribución empírica de frecuencia de los distintos tipos de eje en función de su carga, que se acepta como representativa de la composición del tráfico pesado en un tramo e instante determinado, y se suele generalizar a otros instantes (horas, días, estaciones, años...) (fig. 8).

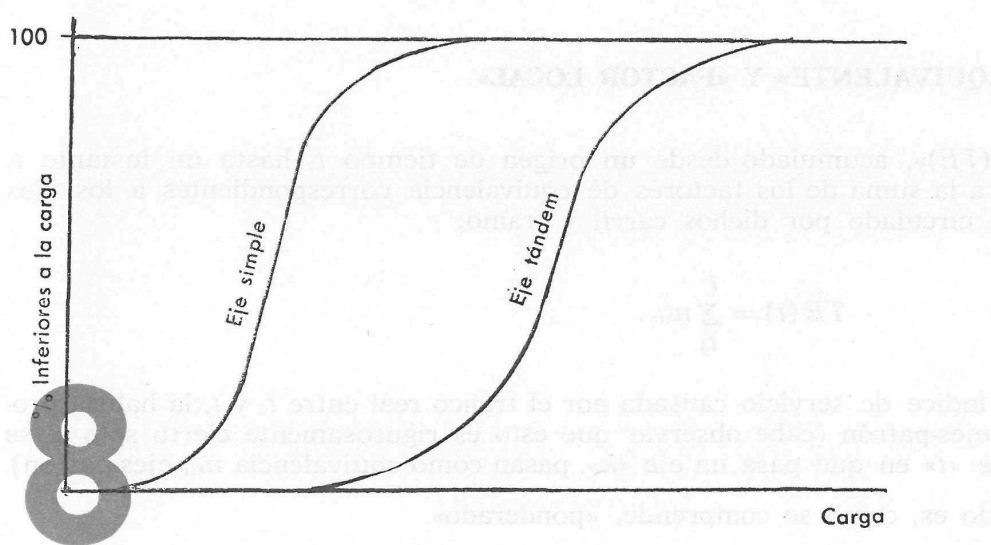

\subsection{CONCEPTO DE «EQUIVA- LENCIA DE EJES»}

Volviendo al esquema indicado en el apartado 4.2 , ya se ha visto en 4.3 la gran diversidad de tipos de cargas de ejes $n_{i}$ que transitan por un tramo determinado. Para poder manejar una sola cifra representativa de la acción del tráfico, se ha convenido en definir el concepto de «eje equivalente», según se describe a continuación.

Elegido un «eje-patrón» que sue- 
le hacerse coincidir con el eje simple cuya carga sea la máxima legalmente permitida (en España $10 \mathrm{t}$; las ruedas, naturalmente, para dichas cargas son gemelas), se compara la disminución del índice de servicio $\Delta p^{p}{ }_{i t}$ que un eje-patrón produciría en el firme «i» e instante «t», con la disminución $\Delta p^{k}{ }_{i t}$ que produce realmente un eje «k» en las mismas circunstancias. Se dice que el paso del eje «k» equivale a

$$
n_{k i t}=\Delta p^{k}{ }_{i t} / \Delta p^{p_{i t}}
$$

pasadas del eje-patrón.

Es importante observar que lo anterior es únicamente válido a efectos de la disminución del índice de servicio y que, por ejemplo, no significa que la deformación puntual producida en el firme por el eje " $k$ » sea $n_{k i t}$ veces la que produciría el eje-patrón. Indudablemente se podría definir una equivalencia basada en tales o parecidas consideraciones, pero no es interesante aquí.

El factor de equivalencia $n_{k i t}$ depende, principalmente:

- del tipo y carga del eje $k$;

— de las condiciones estructurales del firme «i» del tramo en el instante «t».

\subsection{CONCEPTO DE \&TRAFICO PONDERADO»}

La variabilidad de las condiciones estructurales del firme con las circunstancias ecológicas hace que el factor de equivalencia $n_{k i t}$ de un determinado eje " $k$ " sea variable en el tiempo "t» según dichas circunstancias, además de ser distinto de firme a firme («i»).

Una forma conveniente de expresar $n_{k i t}$ es la siguiente:

$$
n_{k i t}=n^{*} \times f(i, t),
$$

en la que:

$n^{*}{ }_{k}=$ el factor de equivalencia del eje $k$, en unas condiciones estructurales $\left(i_{n}, t_{n}\right)$ normalizadas.

$f(i, t)=$ un factor ponderal que representa la variación de las características estructurales entre diversos firmes, y entre las condiciones de un mismo firme según las circunstancias ecológicas. Por definición:

$$
f\left(i_{n}, t_{n}\right)=1
$$

La ventaja de este artificio es evidente si se admite que $f(i, t)$ es independiente del eje «k» a que se refiere (es decir, se puede sacar factor común de todo el tráfico), y que además se puede determinar independientemente.

Las equivalencias de tráfico basadas en $n^{*}{ }_{k}$ se denominan "sin ponderar», y las basadas en $n_{k i t}$ se llaman "ponderadas». En estas últimas quedan englobadas la influencia del tipo de firme y del clima.

\subsection{CONCEPTOS DE «TRAFICO EQUIVALENTE» $Y$ «FACTOR LOCAL»}

Se denomina "tráfico equivalente $(T E)$ », acumulado desde un origen de tiempo $t_{0}$ hasta un instante $t$, y referido a un carril de un tramo, a la suma de los factores de equivalencia correspondientes a los ejes de los distintos vehículos que han circulado por dichos carril y tramo:

$$
T E(t)=\sum_{t_{0}}^{t} n_{k i t}
$$

Por definición, la disminución del índice de servicio causada por el tráfico real entre $t_{0}$ y $t$, la habría producido también el tránsito de $T E$ ejes-patrón (cabe observar que esto es rigurosamente cierto sólo si se considera que en el mismo instante "t» en que pasa un eje «k», pasan como equivalencia $n_{k i t}$ ejes-patrón).

El tráfico equivalente arriba definido es, como se comprende, "ponderado». 
Sin embargo, si se define el efactor ponderal global»

$$
F_{i t}=\frac{\sum_{t_{0}}^{t} n_{k i t}}{\sum_{t_{0}}^{t} n^{*_{k}}}=\frac{\sum_{t_{0}}^{t} n^{*} \cdot f(i, t)}{\sum_{t_{0}}^{t} n^{*}{ }_{k}},
$$

se puede expresar el «tráfico equivalente ponderado $T E$ » en función del «tráfico equivalente sin ponde$\operatorname{rar} T E^{*}$ :

$$
T E=F_{i t} \cdot T E^{*}
$$

Esta descomposición es conveniente porque:

$F_{i t}$ no depende de «k», varía poco con «t», y se puede considerar constante para un tipo de tráfico y un tramo dados («factor local»);

$T E^{*}$ puede determinarse a partir de los estudios de intensidad y composición del tráfico pesado. En efecto, por definición:

$$
T E^{\star}=\sum_{t_{0}}^{t} n^{*}{ }_{k} .
$$

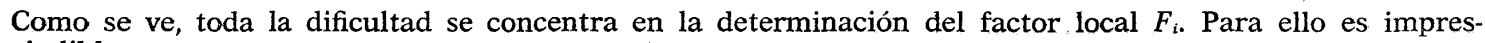
cindible que se conozcan:

- la relación entre las condiciones estructurales del firme y la disminución del índice de servicio causada por el paso de un eje «k»;

- la variación de las condiciones estructurales del firme a lo largo del tiempo.

Estos dos aspectos fundamentales del problema se estudian más adelante.

\section{Cincumstancius ecoldgiterus}

Las circunstancias ecológicas que pueden tener influencia en la capacidad resistente de un firme son, principalmente, las siguientes:

- la temperatura ambiente, que, combinada con la insolación, produce variaciones de temperatura en las capas del firme. La viscosidad de los ligantes bituminosos se modifica con la temperatura y, por consiguiente, también se modifican las propiedades reológicas de las capas que están aglutinadas por dichos ligantes;

- la precipitación de lluvia y la evapotranspiración producen unas variaciones en el estado de humedad de las distintas capas del firme, cuyo efecto en la capacidad resistente es especialmente acusado en la explanada. El fenómeno puede complicarse por la presencia de aguas freáticas.

En los datos relativos a la variación de los factores ecológicos con el tiempo, cabe distinguir una doble faceta: la evolución real, únicamente estudiable para épocas pasadas; y la evolución previsible, extrapolación estadística de largas series de observaciones de la evolución real, que permite prever con cierta exactitud los datos futuros, bien entendido que la realidad irá manifestando discrepancias con la previsión. 

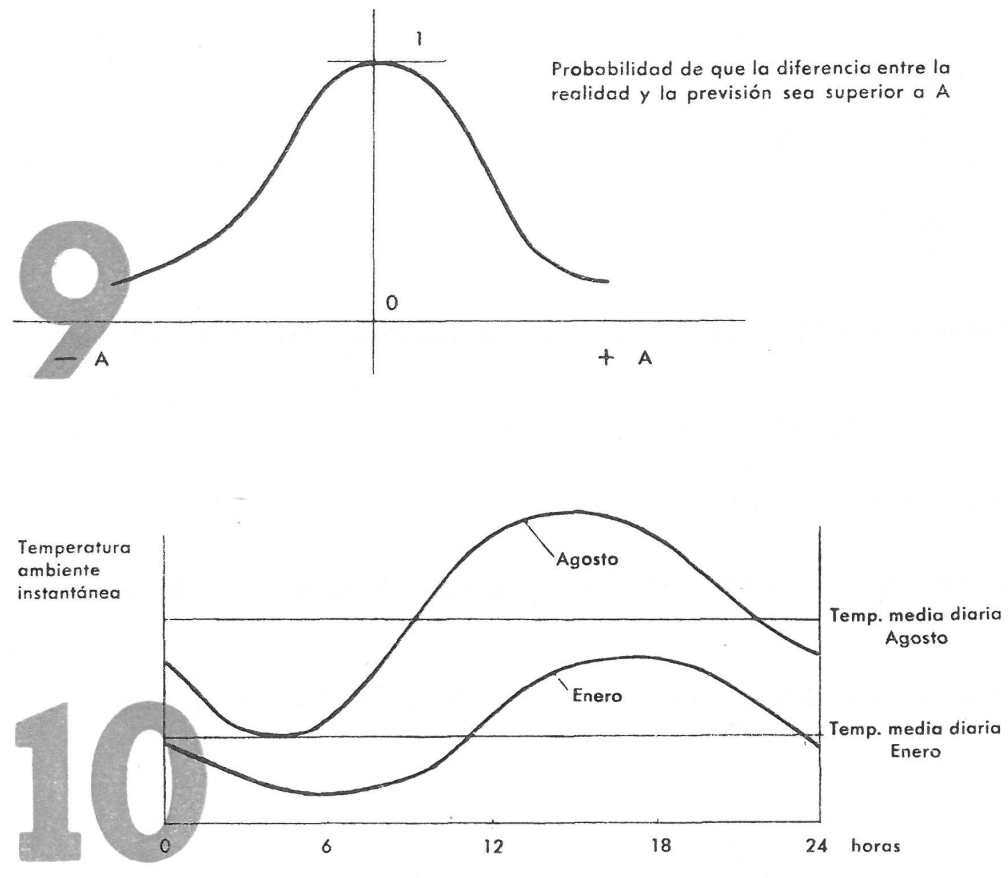

Estas discrepancias tienen una naturaleza estocástica, pudiéndose estudiar la probabilidad de que su magnitud, en un instante determinado, rebase un cierto valor (fig. 9).

\subsection{VARIACIONES DE LA TEMPERATURA AMBIENTE}

La temperatura ambiente se ve sometida a una doble oscilación: diaria y estacional.

La temperatura instantánea oscila alrededor de la media diaria (fig. 10), mientras que, a su vez, la temperatura media diaria oscila alrededor de la media anual (fig. 11).

Es posible que las características (amplitud y fase) de la oscilación diaria estén sometidas también a una oscilación estacional. También existen, indudablemente, variaciones hiperanuales (entre años sucesivos) en los distintos parámetros definitorios de la variación de la temperatura ambiente (temperatura media anual, amplitud y fase de la oscilación anual, amplitud y fase de la oscilación diaria); pero su definición «a priori» es imposible.

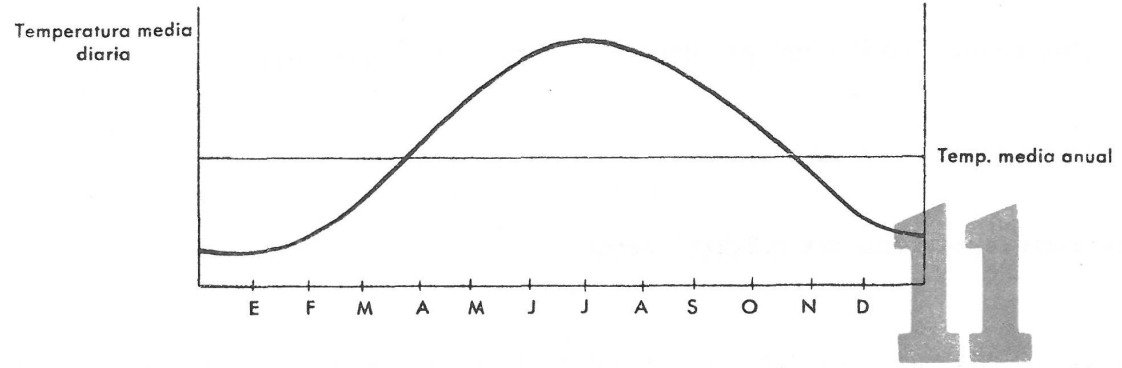

\subsection{INSOLACION}

Por efecto de la energía solar, los pavimentos oscuros (y en especial los bituminosos) pueden adquirir una temperatura superficial muy superior a la ambiente. Siendo prácticamente constante la energía radiada por el sol, la energía recibida por el pavimento en un instante dado depende de la orientación de aquél en dicho instante, y de la eventual presencia de nubosidades que disminuyan o incluso anulen la transmisión.

Por otro lado, si el pavimento tiene una temperatura distinta de la del aire en contacto con él, tenderá a intercambiar con él energía calorífica, hasta que la energía que recibe por insolación equilibre a la perdida por radiación y convección, dependiendo este punto de equilibrio de las características térmicas del material del pavimento. 
El estudio termotécnico de estos intercambios de calor es perfectamente posible, con la única incertidumbre de los datos de insolación y temperatura ambiente en cada instante. Pero si se conoce la ley de variación de estos datos con el tiempo, bien sea experimentalmente (años transcurridos), bien sea como previsión estadística (años futuros), se puede conocer la ley de variación de la temperatura superficial del pavimento a lo largo del tiempo considerado.

\subsection{PRECIPITACION}

Basándose en los datos meteorológicos es posible conocer la variación de la precipitación acumulada a lo largo del año, ya sea éste real (años transcurridos) o bien teórico (años medios previsibles) (fig. 12).

No es necesario un análisis detallado de la repartición temporal de esta precipitación, como sería el caso para el estudio de desaguies: pues su repercusión en la humedad de la explanada es más bien lenta.

La precipitación se traduce en un aumento de la humedad de la explanada, en cuya cuantía influyen la duración de la lluvia, la escorrentía superficial, la capacidad de infiltración de las capas superiores, el estado de compactación y de humedad existente en la explanada, y la presencia de agua freática.

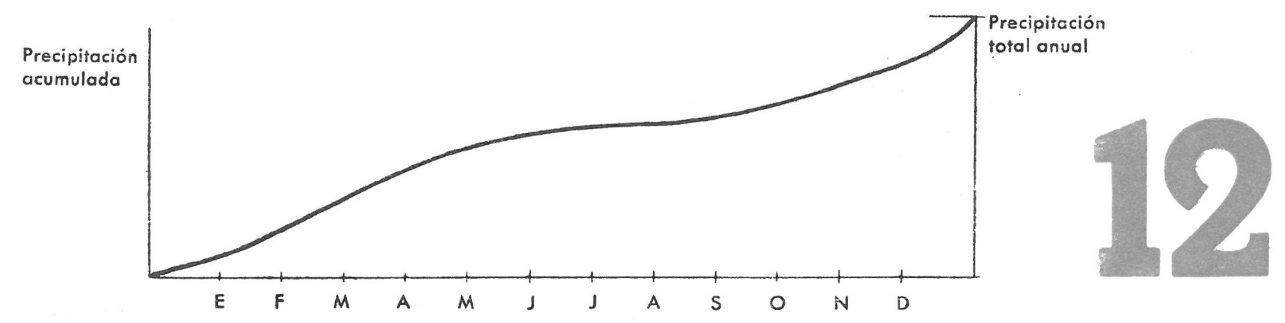

\subsection{EVAPOTRANSPIRACION}

El agua contenida en el terreno, y concretamente en la explanada, tiende a eliminarse hacia la atmósfera (más seca), infiltrándose hacia la superficie y allí evaporándose. En el fenómeno influyen:

- la temperatura y humedad atmosféricas, así como la presencia de viento;

- la textura de la superficie que evapora, y la insolación que recibe;

- la permeabilidad y capacidad de retención de agua de las capas del firme y de la explanada.

El estudio del fenómeno por un método racional es muy complejo, por lo que se recurre generalmente a la aplicación de las fórmulas empíricas, establecidas por lo común con fines agronómicos, o a la experimentación mediante lisímetros. En la explanada, la evaporación directa se suele ver coartada por la impermeabilidad de las capas superpuestas (pavimento y arcenes), y facilitada por la presencia de drenajes.

\subsection{BALANCE HIDRICO}

La acción de la precipitación y de la evapotranspiración se combinan y contrarrestan, resultando un saldo de humedad en el terreno, que se denomina balance hídrico del mismo. La humedad oscila entre la higroscópica (mínima no eliminable) y la de saturación (máxima, que depende de la compactación del terreno) (fig. 13).

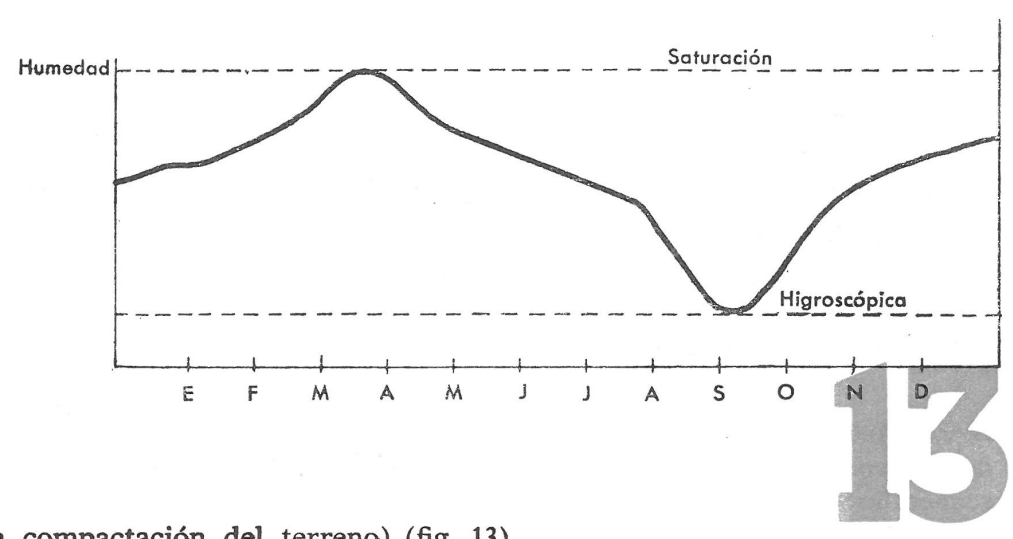

(Continuará en el próximo número). 


\section{résumed \\ Bases d'une méthode rationnelle pour le dimensionnement des renforce- ments de revêtements souples, basée sur la mesure de la déflexion sous axe chargé}

Sandro Rocci, ingénieur des Ponts et Chaussées

Cet article est une description qualitative des facteurs intervenant dans le comportement du revêtement routier et des rapports existant entre eux. Un schéma fonctionnel en est établi, dont l'objectif ultime économique est la réduction au minimum du coût total (exécution + entretien + exploitation) d'une route. Sont également établies les bases pour la création d'une méthode de dimensionnement des renforcements pour les revêtements soples, dont la justification quantitative sera l'objet d'un prochain article.

\section{Basic data to define a national system of designing overlays of flexible pavements, based on deflection under a loaded axle}

Sandro Rocci, civil engineer

This article gives a qualitative description of the factors that influence in the behaviour of a road pavement, and of the relations between these factors. A functional pattern is established, whose final economic aim is the reduction of the overall cost (construction plus maintenance plus exploitation) of a road. Further, principles are established for the design of overlays for flexible pavements: the quantitative justification of these criteria will be discussed in another article.

\section{Grundlagen einer rationalen Methode für die Dimensionierung von Beans- pruchungen von schmiegsamen Decken, die auf der Messung der Punktver- formung unter einer belasteten Achse gegründet wird}

Sandro Rocci, Bauingenieur

Dieser Artikel stellt eine qualitative Beschreibung der Faktoren, die in der Verhaltung der Strassendecken vorkommen, und der Beziehungen unter ihnen dar. Man setzt ein Schema fest, dessen letzten economischen Zweck die minimalen Gesamtkosten einer Strasse (Bau, Aufbewahrung und Betrieb) 1st. Gleichzeitig setzt man die Grunlagen für eine Dimensionierungsmethode von Beanspruchung von schmiegsamen Decken fest, die auf der Messung der Punktverformung unter einer belasteten Achse gegruindet wird; der quantitative Nachweis davon wird das Thema für den nächsten Artikel sein. 